\title{
TECHNIQUE
}

\section{Combined mechanical and thrombolytic treatment for totally occluded bypass grafts}

\author{
C Lotan, M Mosseri, Y Rozenman, Y Hasin, M S Gotsman
}

\begin{abstract}
Totally occluded venous grafts are usually less amenable to mechanical reperfusion alone (for example percutaneous transluminal coronary angioplasty, PTCA) because of the large mass of thrombotic material within the graft. A combined approach using mechanical and thrombolytic treatment might therefore be more successful. Twenty one patients (20 males, one female) with a mean age of 64.5 (SD 5.6) years underwent angiography because of crescendo or unstable angina $(n=19)$ or myocardial infarction $(n=2)$ at a mean of $21 \cdot 7$ (18.6) days after onset of symptoms (range 1-60). All patients had had coronary artery bypass grafting (CABG) at a mean of 8.02 (4.02) years (range 0.3-13 years) before the current admission. At catheterisation, totally occluded venous bypass grafts to the left anterior descending coronary artery or diagonal $(n=10)$, marginal $(n=6)$, or right coronary artery $(n=5)$ were found. A combination of PTCA and thrombolytic treatment (in eight patients extended thrombolysis for 24 hours) was successful in reopening the venous graft in $16 / 21$ patients $(76 \cdot 2 \%)$. Immediate complications included femoral haematoma (4), distal embolisation (3), and infection in one patient. Out of 13 patients catheterised within three months, two had reoccluded, seven had restenosis, while four had patent grafts. Recurrent PTCA (at least once more) was done in eight patients. At long term follow up of a mean of 26.7 (21.6) months (range 4-75 months), four patients were asymptomatic, eight still suffered from mild stable angina, while three had recurrent hospital admissions and needed a second coronary artery bypass. A combination of thrombolytic treatment and PTCA is a feasible and practised approach to recanalise recently occluded venous bypass grafts.
\end{abstract}

(Br Heart f 1994;74:455-459)

Keywords: percutaneous transluminal coronary angioplasty; thrombolytic treatment; coronary artery bypass; venous bypass graft occlusion
Late occlusion of coronary artery bypass grafts usually presents as new or worsening angina pectoris and often as acute myocardial infarction. In most patients, the occluded graft is filled with thrombotic material so that conventional thrombolysis is relatively ineffective in restoring good flow in the graft. ${ }^{2}$

Conventional percutaneous transluminal coronary angioplasty (PTCA) also has a low success rate and may result in extensive distal embolisation. ${ }^{3}{ }^{4}$ This study will examine the potential of using a combination of mechanical and thrombolytic treatment for totally occluded bypass grafts.

\section{Methods}

Between 1987 and 1992, 3416 PTCA procedures were performed at the Hadassah University Hospital catheterisation laboratory, of which $125(3.7 \%)$ were performed on coronary venous bypass grafts. In 21 of these patients $(16 \cdot 8 \%)$ the venous bypass graft was found to be totally occluded at the time of angiography, but the occlusion was judged to be recent on the basis of clinical information (recent onset of symptoms) and angiographic findings (defined deep niche $>1 \mathrm{~cm}$ and/or staining of a thrombus in the graft). Therefore in these cases a combination of PTCA and intragraft thrombolysis was attempted to reopen the occluded graft. In 13 patients, repeat angiogram was performed between two and six months after initial PTCA to determine the long term results.

All cineangiograms were reviewed by two observers to determine the distance from graft orifice to occlusion, the pattern of retrograde filling of the culprit artery, the existence of thrombotic material, and the primary and long term success.

\section{PTCA PROCEDURE}

In all patients, an initial attempt was made to pass a PTCA guiding wire through the graft into the distal coronary artery. This was done in order to assess the freshness of the thrombus and to facilitate delivery of thrombolytics into the graft. This was followed by slow intragraft infusion of urokinase (a dose ranging from 500000 to 1.5 million) or streptokinase at a dose of 750000 units. After 30-60 minutes an attempt was made to pass a 1.5 $\mathrm{mm}$ balloon catheter through the graft with 
Table 1 Complications after PTCA to graft

\begin{tabular}{lr}
\hline Cardiac \\
\multicolumn{2}{l}{ Myocardial infarction } \\
+ ventricular fibrillation 1 \\
CK increase & 3 \\
General & \\
Femoral haematoma & 4 \\
Fever, infection & 3 \\
\hline CK, creatine kinase.
\end{tabular}

Table 2 Short term results after successful PTCA $(n=13)$

\begin{tabular}{ll}
\hline Patent grafts & 4 \\
Restenosis & 7 \\
Reocclusion & 2 \\
\hline
\end{tabular}

recurrent dilatation distally to facilitate the delivery of thrombolytics into the thrombus. Improvement in recanalisation was assessed by repeated injection of contrast material as the balloon was advanced distally. In patients in whom an open channel was achieved and distal flow in the native coronary artery observed, a balloon with a larger diameter was used to dilate the stenotic segment simultaneously with the continuous infusion of the thrombolytic agent. In patients who had a large thrombotic mass, a guiding or diagnostic catheter was left in the graft orifice and continuous urokinase at a rate of 100000 units/hour was delivered into the graft through an infusion pump for a further 24 hours, the procedure being completed the following day.

All patients were maintained on intravenous heparin (to keep their partial thromboplastin time at twice normal) for 3-5 days, overlapping with adequate oral anticoagulant therapy. In addition, the patients received aspirin, nitrates, and calcium channel blockers.

Values are given as mean (SD).

\section{Results}

PATIENT CHARACTERISTICS

Twenty one patients were included in the study: $20(95 \%)$ were males and the mean age was $64.5(5.6)$ years. The mean time interval from coronary artery bypass grafting to the current catheterisation was $8.02(4.02)$ years (range $1-13$ years). The mean number of bypass grafts was $2 \cdot 28(1.02)$ grafts per patient.

The presenting symptoms were crescendo or unstable angina pectoris in 19 patients and myocardial infarction in two. The mean time from onset of symptoms to catheterisation was $21.7(18.6)$ days with a range of $1-60$ days. None of the patients received thrombolytic treatment before coronary catheterisation.

ANGIOGRAPHIC FINDINGS AND PTCA RESULTS Out of a total of 49 venous bypass grafts in the entire group, only 12 grafts $(24.4 \%)$ were still patent at the time of angiography. In 21 of the 37 occluded grafts, the occlusion was judged to be recent.

The culprit occluded venous graft supplied the left anterior descending coronary artery or a diagonal branch in 10 patients, the first or second marginal artery in six patients, and the distal right coronary artery in five patients. In 15 patients $(71 \%)$ a well defined niche $(>1$ $\mathrm{cm}$ in length) from graft orifice to occlusion was observed. In 20 of the vein grafts, the thrombus was extensive ( $>3 \mathrm{~cm}$ in length) and filled the entire graft.

Interestingly, in only five of the patients could flow in the native coronary artery supplied by the occluded grafts be seen. In three patients poor anterograde flow was seen with diffuse severe proximal disease, while in two others marginal and posterior descending coronary arteries were retrogradely filled through a distal left anterior descending artery.

The combined strategy restored flow in 16 of the 21 patients $(76 \%)$. In two patients, the PTCA wire could not be passed through the graft and thrombolysis did not restore flow. In three other patients, the PTCA guide wire could be passed through the graft but the thrombotic mass was very large, and an open channel could not be achieved despite prolonged (up to 24 hours) thrombolytic therapy. The mean time from coronary artery bypass graft in these five patients $(7 \cdot 7(2 \cdot 8)$ years) was not different from the entire group $(8.02(4.02)$ years). However, the time from symptom onset to coronary catheterisation in these five patients was 41 (22) days compared to 14.3 (15) days for the remaining patients. Of the five patients in whom visible run off could be seen in the native coronary arteries, revascularisation was successful in only two.

In most patients (13 of 16 successfully opened grafts) signs of residual thrombus were noted (hazy areas, small filling defects, and irregular luminal surface) and thus heparin treatment was continued in all patients for at least 72 hours, overlapping and continuing with oral anticoagulant therapy.

Procedural complications occurred in 11 patients (table 1). One patient developed ventricular fibrillation during infusion of urokinase to a proximally occluded left anterior descending coronary artery graft, and intraaortic balloon pump support was needed. This patient subsequently developed acute anterior myocardial infarction. Three other patients had a rise in creatine kinase to 321 (192) units without significant changes on the electrocardiogram (for example, new $Q$ waves), probably reflecting distal embolisation. Four patients had a large femoral haematoma and needed blood transfusion, and three patients with prolonged thrombolytic therapy developed fever and chills requiring antibiotic treatment.

Short term results are listed in table 2 . Of the 16 patients with successful procedures, 13 (81\%) were catheterised within two to four months after PTCA. In four patients, the graft was patent, in seven graft restenosis occurred, while in two the graft reoccluded. Eight of these patients had one or more PTCA attempts.

At long term follow up (mean 26.7 (21.6), range 4-75 months), six patients were free of angina, eight had mild angina (grades I or II according to Canadian Cardiovascular Society function classification), while three had recurrent hospital admissions and underwent a second coronary artery bypass graft.

The figure shows sequential saphenous vein graft angiograms from a patient with an occluded graft to the right coronary artery.

\section{Discussion}

Recent studies have shown that symptomatic late graft occlusion is caused by thrombotic occlusion with a pathological process that is similar to native coronary artery occlusion. ${ }^{1-3}$ 
Sequential saphenous vein graft angiograms from patients with an occluded graft to the right coronary artery. (A) $A 10 \mathrm{~mm}$ niche of the occluded graft to the right coronary artery (arrow; left anterior

oblique view). (B) $A$ high torque floppy guiding wire through the graft into the distal right coronary artery. (C) Injection of contrast during urokinase infusion. Partial filling of the graft with staining (arrow) compatible with a large thrombotic mass. (D) 24 hours after continuous urokinase infusion. There is still haziness and stenotic segments of the grafts. (E) Inflation of a long balloon (40 $\mathrm{mm}$ length) in the graft. (F) Final appearance of the grafi with good flow to the distal artery.
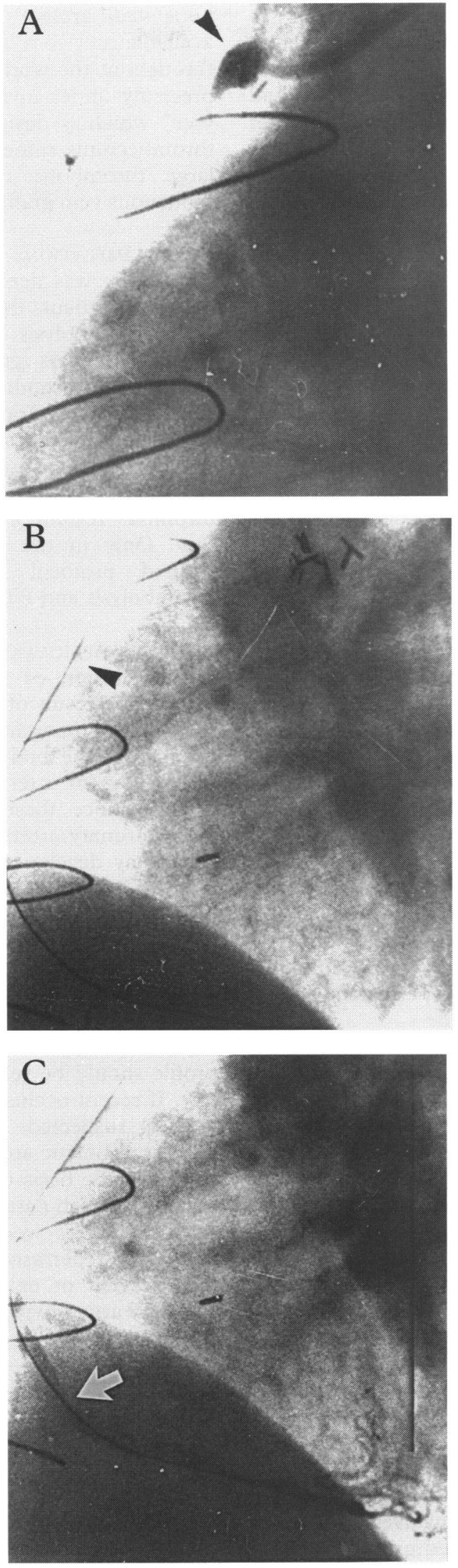

The absence of side branches and distal run off in the graft leads to stagnation of blood with rapid proximal propagation of the thrombus, forming a large thrombotic mass. This explains the relatively low success of conventional intravenous thrombolysis in restoring coronary flow. Similarly, conventional balloon techniques were found to be inadequate to deal with the large mass of
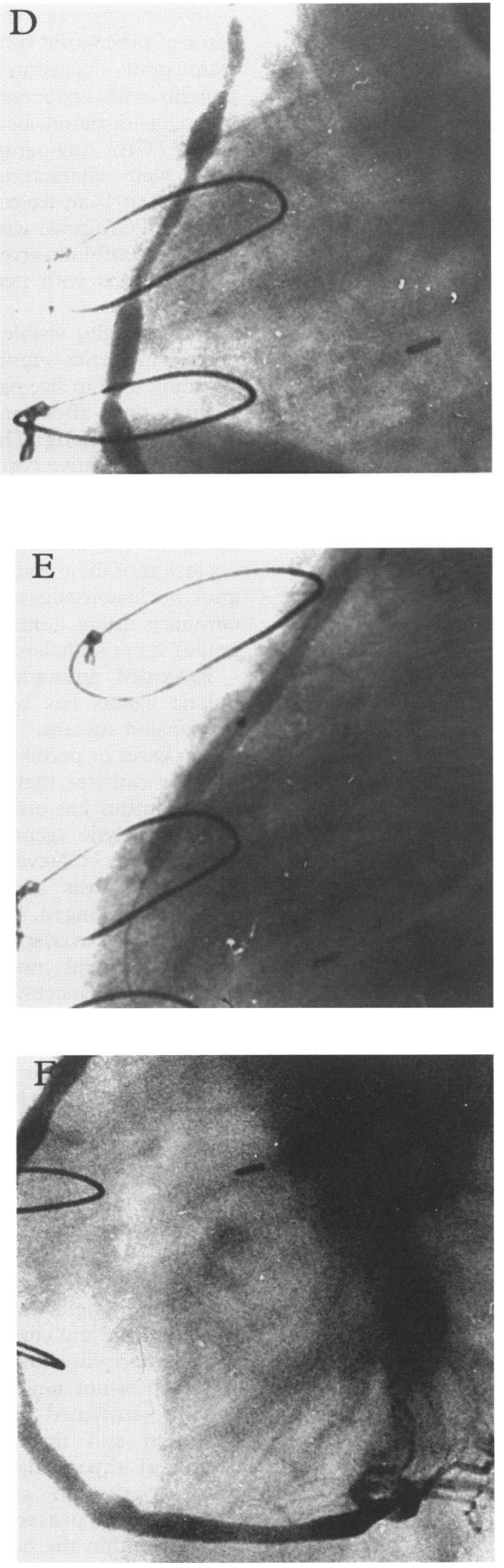

thrombotic material, and the danger of distal embolisation was very high. ${ }^{2}$ Thus an attempt to combine both mechanical and intragraft thrombolysis may be more successful in restoring coronary blood flow. Indeed, using this approach, a relatively high initial success rate $(76 \cdot 2 \%)$ for restoring graft patency was achieved, similar to the findings reported by Hartmann et al. ${ }^{4}$ 
In our experience the most important indicator of procedural success was the time lapse from graft occlusion to PTCA. The five patients with unsuccessful recanalisation had a longer duration between symptom onset and PTCA. Angiographically, recent occlusions were characterised by a deep niche (more than $1 \mathrm{~cm}$ from graft orifice), ability to pass a PTCA guide wire, and the existence of recent thrombi observed as circular lesions or filling defects with persistent contrast staining.

Interestingly, visible run off in the native coronary arteries supplied by occluded grafts was only seen in five patients. It might be that a short time after graft occlusion collateral flow is still lacking. Thus a visible retrograde filling of the native coronary arteries may suggest a more chronic process. Alternatively, it might be that patients with good retrograde filling of the coronaries may have "silent" occlusion of their grafts, while "symptomatic" graft occlusion suggests that the flow in the coronary artery (either antegrade or retrograde) is not sufficient.

Extended intragraft delivery of thrombolytic agents has been shown to improve reperfusion success. ${ }^{5-7}$ The use of special perfusion wires or perfusion catheters such as the Tracker catheter that can be placed deep in the thrombus has enabled improved delivery of thrombolytic agents with better resolution of thrombus. ${ }^{38}$ Nevertheless, in most cases residual stenosis and thrombus still exist. Thus a prolonged infusion of intravenous heparin with overlapping to oral anticoagulants for several months is essential in all cases where successful reperfusion of an occluded graft was achieved.

Despite the fact that the overall short term restenosis/reocclusion rates found in this study were relatively high, they are not significantly different from restenosis rates in patients undergoing PTCA in the shaft of a saphenous bypass graft. However, since in most of these cases only restenosis occurred, successful repeat PTCA was achieved in eight patients $(87 \%)$. Long term results showed that the majority of patients had either minimal or no angina at all, while only three required a second coronary artery bypass.

The morbidity involved in this combined approach is not negligible, since one of our patients sustained a $Q$ wave myocardial infarction and three others showed some degree of myocardial damage sufficient to cause a rise in creatine kinase values. Moreover, this procedure involves leaving the catheter within the occluded graft for a relatively prolonged period of thrombolysis treatment, thus increasing the risk of infection. Indeed, signs of infection and femoral haematomas were seen in $14.2 \%$ and $19 \%$ of the patients respectively.

Therefore a search for new devices that can achieve better removal of the thrombotic material must continue. One of these devices is the transluminal extraction atherectomy (TEC) ${ }^{9}$ which has been specifically recommended for dealing with degenerated saphe- nous vein grafts. ${ }^{10}$ The TEC, however, is available only to a limited number of centres throughout the world. Another device that is presently under investigation is the "hydrolyser" which is designed as a hydrodynamic thrombectomy catheter to deal especially with large thrombotic masses in old diseased saphenous vein grafts. ${ }^{11}$

\section{STUDY LIMITATIONS}

This study was derived from a retrospective cohort of patients that had a combined intragraft thrombolysis and PTCA for totally occluded bypass grafts. Since no uniform approach to thrombolysis was used, the dose and length of intragraft thrombolytic treatment varied, and were dependent primarily upon operation assessment of thrombotic mass, as well as on the initial degree of thrombus resolution in the catheter laboratory. Only in the last five patients was a defined protocol of extended intragraft thrombolysis and PTCA used.

\section{CLINICAL IMPLICATIONS}

Late occlusion of saphenous vein grafts is usually the result of acute thrombotic occlusion of underlying fibrotic and atherosclerotic changes in the body of the graft as well as progression of arteriosclerosis in the grafted vessels. Since these patients usually suffer from coronary artery disease for many years, most may develop some degree of collateral flow as well as progression of arteriosclerosis in the grafted vessels. Thus most patients with graft occlusion may experience only mild changes in the anginal syndrome and only a few develop myocardial infarction. Therefore patients after coronary bypass grafts who experience significant changes in their anginal profile should be referred for early angiography. If recent occlusion of the venous bypass graft is suspected, a combined mechanical and thrombolytic approach should be considered. On the basis of the exact angiographic findings in each patient, the risk of the procedure should be weighed against the benefit that early reperfusion of the occluded graft may prevent or delay the need for another coronary artery bypass graft.

We thank Chava-Ita Kaniel for helping in data collection and preparation of the manuscript and Shoshanna Weinstein for secretarial assistance.

1 Grines CL, Booth DC, Nissen SE, Gurley JC, Bennett KA, O'Connor WN, et al. Mechanism of acute myocardial infarction in patients with prior coronary artery dial infarction in patients with prior coronary artery bypass grafting and the
Cardiol 1990;65:1292-6.

2 Sabri MN, Johnson D, Warner M, Cowley MJ. Intracoronary thrombolysis followed by directional atherectomy: a combined approach for thrombotic vein graft lesions considered unsuitable for angioplasty. Cathet Cardiovasc Diagn 1992;26:15-8.

3 Bell C, Kern MJ, Kaiser G. Sequential proximal and distal infusion of urokinase resulting in recanalization of acutely occluded aortocoronary bypass graft after coronary angioplasty. Cathet Cardiovasc Diagn 1992;26: $224-8$. 4 Hartmann JR, McKeever LS, Stamato NJ, Bufalino VJ, cally occluded aortocoronary saphenous vein bypass grafts by extended infusion of urokinase: initial results and short term clinical follow up. $尹$ Am Coll Cardiol 1991;18:1517-23. 
5 Mosseri $M$, Hasin $Y$, Admon $D$, Gotsman MS Thrombolytic therapy of a stenosed coronary vein bypass graft with continuous intragraft streptokinase drip. Cardiology 1990;77:318-21

6 Chapekis AT, George BS, Candela RI. Rapid thrombus dissolution by continuous infusion of urokinase through an intracoronary perfusion wire prior to and following PTCA: results in native coronaries and patent saphenous vein grafts. Cathet Cardiovasc Diagn 1991;23: 89-92.

7 Doorey AJ, Rosenbloom MA, Zolnick MR. Successful angioplasty of a chronically occluded saphenous vein graft using a prolonged urokinase infusion from the brachial route. Cathet Cardiovasc Diagn 1991;23:127-9.
8 Hibbard MD, Holmes DR. The Tracker catheter: a new vascular access system. Cathet Cardiovasc Diagn 1992; 27:309-16.

9 Popma J, Leon MB, Mintz OS, Kent KM, Satter LF, Oarraind TJ, et al. Results of coronary angioplasty usin the transluminal extraction catheter. Am $f$ Cardio 1992;70:1526-632.

10 Sketch MH, Labinaz M, Stack S. Extraction atherectomy. In: Topol A. Textbook of interventional cardiology, 2nd ed. Philadelphia: WB Saunders, 1994:668-77.

11 Fajadet J, Diaz L, Cassagneau B, Robert G, Bar O, Marco J. The hydrodynamic thrombectomy catheter: preliminary experience in human old saphenous vein grafts [abstr]. Circulation 1993;88:I-64. 